\title{
Metabolic Control of Circulation
}

\author{
EFFECTS OF IODOACETATE AND FLUOROACETATE
}

\begin{abstract}
Chang-SEng Liang
From the Departments of Medicine and Pharmacology, and the Cardiovascular Institute, Boston University School of Medicine, the Cardiology Department, the Medical Service and Thorndike Memorial Laboratories, Boston City Hospital, and the Departments of Medicine and Clinical Research, University Hospital, Boston, Massachusetts 02118
\end{abstract}

\begin{abstract}
A B S TRACT The circulatory effects of selective metabolic inhibition of glycolysis and of the tricarboxylic acid cycle by iodoacetate and fluoroacetate were studied in intact chloralose-anesthetized dogs. Pulmonary arterial blood pressure and vascular resistance increased after administration of both inhibitors, but neither systemic hemodynamics nor myocardial contractility changed significantly. Coronary blood flow did not change after iodoacetate administration but increased four- to fivefold after fluoroacetate. Administration of normal saline had no effect on any of the parameters. The changes in pulmonary arterial blood pressure and coronary blood flow after fluoroacetate were not mediated via the autonomic nerves or adrenergic neurohumors because they still occurred after autonomic nervous system inhibition. Neither myocardial oxygen consumption nor left ventricular work changed. A selective increase in myocardial blood flow also occurred in conscious dogs after fluoroacetate administration; hepatic artery flow was reduced, but other organ flows did not change significantly. These results indicate that pulmonary pressor and coronary dilator effects may be produced in intact dogs by selective metabolic blockade, in the absence of reduced oxygen supply or impairment in the electron transport system. These results also suggest that the increases in pulmonary arterial blood pressure, coronary blood flow, and cardiac output that occur during hypoxia probably are related to separate metabolic events in the tissue.
\end{abstract}

This work was presented in part before the 49th Scientific Session of the American Heart Association in Miami Beach, Fla. 16 November 1976, and appeared in Circulation. 54 (Suppl. II): II-107.

Received for publication 23 August 1976 and in revised form 17 March 1977.

\section{INTRODUCTION}

The increases in cardiac output and coronary blood flow in intact animals which occur during arterial hypoxemia are associated not only with a reduction in tissue oxygen tension but also with a variety of metabolic changes. Cardiac output and coronary blood flow also may increase in the absence of any reduction in tissue oxygen tension when metabolic changes like those occurring in hypoxia are produced by cyanide $(1,2)$. Thus, these hemodynamic changes could be attributed, at least in part, to alterations in metabolite contents typical of hypoxia. The metabolites or portions of the metabolic systems that initiate the circulatory stimulation, however, are not known, partly because virtually all tissue metabolic processes are affected by the inhibitory actions of hypoxia and cyanide on the electron transport system. In the present experiments, attempts were made to produce more selective metabolic blockade, to ascertain whether changes in cardiac output and coronary blood flow are associated with alterations in glycolytic and tricarboxylic acid cycle activities.

\section{METHODS}

Adult male dogs weighing 17-31 kg after overnight fast were anesthetized with intravenous chloralose $(60 \mathrm{mg} / \mathrm{kg}$ ) after induction with vaporized halothane (Fluothane, Ayerst Laboratories, New York). The trachea was cannulated with a T-tube connected to a Benedict-Roth spirometer filled with $100 \%$ oxygen to record the rate of oxygen consumption. A femoral artery was cannulated with a French 8 Cournand catheter and the pulmonary artery with a French 7 SwanGanz catheter (Edwards Laboratories, Inc., Santa Ana, Calif.) via the right external jugular vein; both catheters were connected to Statham P23Db pressure transducers and a multichannel Brush 480 recorder (Gould, Inc., Instrument Systems 


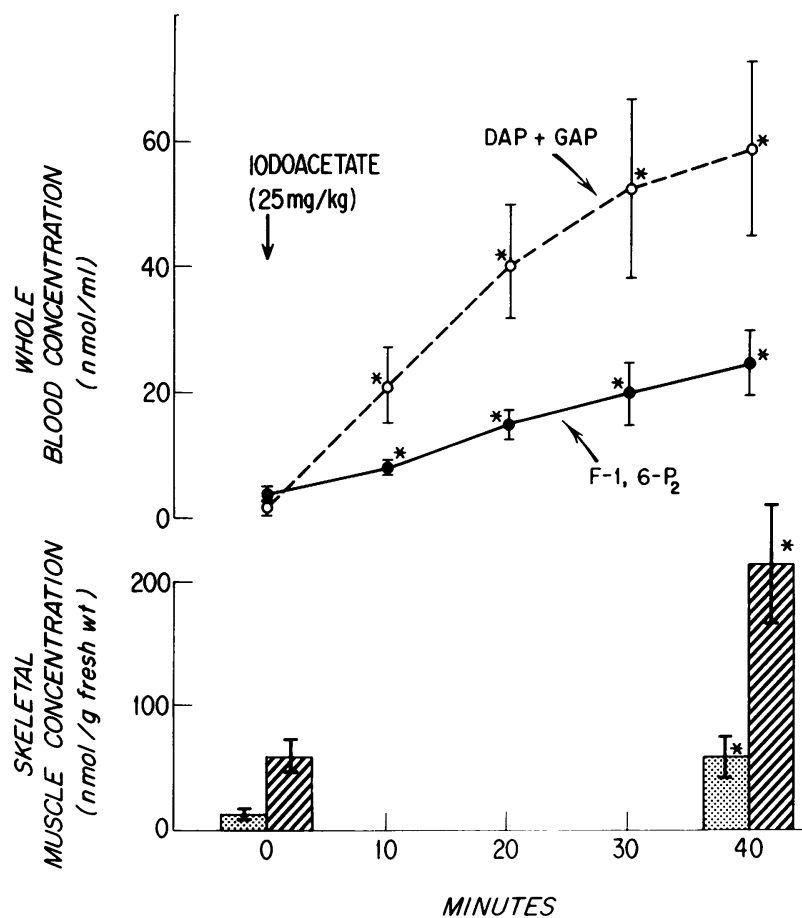

FIGURE 1 Changes in concentrations of fructose-1,6-diphosphate $\left(\mathrm{F}-1,6-\mathrm{P}_{2}\right)$ and triose phosphates (dihydroxyacetone phosphate plus glyceraldehyde-3-phosphate, DAP + GAP) in arterial blood and skeletal muscle after iodoacetate administration $(n=8)$. Vertical lines indicate the means $\pm S E$. Stippled and striped columns indicate DAP + GAP and F-1, $6-\mathrm{P}_{2}$, respectively. Asterisks indicate values that are different from the control at $P<0.05$.

Division, Cleveland, Ohio) to measure systemic and pulmonary arterial blood pressures and heart rate.

The left ventricle was cannulated via the left carotid artery by a Millar transducer-tip catheter (Millar Instruments, Inc., Houston, Tex.) for measuring left ventricular enddiastolic pressure and the first derivative of left ventricular pressure $(\mathrm{dP} / \mathrm{dt})$. The ratio of $\mathrm{dP} / \mathrm{dt}$ to developed pressure $(\mathrm{dP} / \mathrm{dt} / \mathrm{P})$ at a developed left ventricular pressure of $50 \mathrm{~mm}$ $\mathrm{Hg}$ occurring during isovolumic systole was calculated.

Cardiac output was determined by the indocyanine green (Cardio-Green) dye dilution technique (1), using a Gilford Model 140 cardiac output system (Gilford Instrument Laboratories, Inc., Oberlin, Ohio). Systemic and pulmonary vascular resistances were calculated by conventional formulas.

In addition, a catheter was placed fluoroscopically via a jugular vein into the coronary sinus for measuring coronary blood flow, with a 4-aminoantipyrine indicator method $(3,4)$. Diastolic coronary vascular resistance (CVRd), left ventricular work (LVW), myocardial oxygen consumption $\left(\mathrm{MVO}_{2}\right)$, and index of mechanical efficiency (ME) were calculated as follows (5):

$$
\begin{gathered}
\text { CVRd }\left(\text { dyn } \cdot \mathrm{s} \cdot \mathrm{cm}^{-5}\right)=\frac{\text { Adm }- \text { CVm }}{\mathrm{CBF} \times 0.75} \times \mathrm{DFP} \times 1,332 ; \\
\mathrm{LVW}(\mathrm{kg} \cdot \mathrm{m} / \mathrm{min})=\frac{\mathrm{LVsm} \times \mathrm{CO} \times 1.36}{100}
\end{gathered}
$$

$\mathrm{MVO}_{2}(\mathrm{ml} / 100 \mathrm{~g}$ per min $)=\mathrm{CBF} \times\left(\mathrm{CaO}_{2}-\mathrm{CcsO}_{2}\right) \div 1,000$;

$$
\mathrm{ME}(\%)=\frac{\mathrm{LVW}}{\mathrm{MVO}_{2} \times 2.06} ;
$$

where Adm is arterial mean diastolic pressure, $\mathrm{mm} \mathrm{Hg}$; $\mathrm{CVm}$, coronary venous mean pressure, $\mathrm{mm} \mathrm{Hg}$; $\mathrm{CBF}$, coronary blood flow, $\mathrm{ml} / 100 \mathrm{~g} / \mathrm{min}$; DFP, coronary diastolic inflow period, s/min; LVsm, left ventricular systolic mean pressure, $\mathrm{mm}$ $\mathrm{Hg}$; $\mathrm{CO}$, cardiac output, liters/min; $\mathrm{CaO}_{2} ;$ femoral arterial oxygen content, $\mathrm{ml} / \mathrm{liter}$; $\mathrm{CcsO}_{2}$, coronary sinus oxygen content, $\mathrm{ml} /$ liter.

Arterial and coronary sinus venous blood samples were taken for measuring $\mathrm{pH}$ on a Radiometer PHM71 AcidBase Analyzer (The London Co., Westlake, Ohio), $\mathrm{O}_{2}$ content (6), calcium (7), lactate (8), pyruvate (9), glucose (10), and free fatty acids (11). Myocardial extraction coefficients and utilization rates of the substrates were calculated.

Glycolytic inhibition was produced in eight dogs by intravenous infusion of a neutral $5 \%$ sodium iodoacetate solution ( $25 \mathrm{mg} / \mathrm{kg}$, Mann Research Laboratories, Inc., New York) over a 5-min period. Fructose-1,6-diphosphate, glyceraldehyde-3-phosphate, and dihydroxyacetone phosphate (12) were measured in arterial blood samples every $10 \mathrm{~min}$ and in quadriceps muscle biopsy samples taken with rapid freezing technique during the control period and at $40 \mathrm{~min}$ after iodoacetate infusion. Systemic and pulmonary hemodynamic parameters were measured every $5 \mathrm{~min}$ for $40 \mathrm{~min}$, and coronary hemodynamic and metabolic parameters were obtained at 20 and $40 \mathrm{~min}$. These hemodynamic and metakolic parameters also were measured in five dogs after similar administration of normal saline $(0.5 \mathrm{ml} / \mathrm{kg})$.

Sodium fluoroacetate $(2 \mathrm{mg} / \mathrm{kg}$ of a $1 \%$ solution, Sigma Chemical Co., St. Louis, Mo.) was administered intravenously to 14 dogs to inhibit the utilization of citrate. Blood and skeletal muscle citrate concentrations (13) were measured every 10 and every $30 \mathrm{~min}$, respectively, for $1 \mathrm{~h}$. Systemic and pulmonary hemodynamics were measured every $10 \mathrm{~min}$, and coronary hemodynamic and metabolic parameters every $30 \mathrm{~min}$ for $1 \mathrm{~h}$. These measurements were continued for an additional $30 \mathrm{~min}$ in six dogs. Heart muscle samples were taken through left thoracotomy, while the animals were artificially respired, for citrate measurements $60 \mathrm{~min}$ after fluoroacetate administration in seven dogs; cardiac citrate concentration was also measured in five normal dogs.

To eliminate a potential role of autonomic nerves on the hemodynamic effects of fluoroacetate, fluoroacetate $(2 \mathrm{mg} / \mathrm{kg})$ was administered to 10 dogs $30-45 \mathrm{~min}$ after vagotomy and pretreatment with mecamylamine $(5 \mathrm{mg} / \mathrm{kg}$ i.v. $)$ and propranolol $(0.3 \mathrm{mg} / \mathrm{kg}$ i.v. $)$; 5 of them were also pretreated with phentolamine ( $5 \mathrm{mg} / \mathrm{kg}$ i.v.). Ganglionic blockade, car$\operatorname{diac} \beta$-adrenoceptor inhibition, and vascular $\alpha$-adrenoceptor blockade were verified in each dog by comparing the responses to common carotid occlusion, an isoproterenol sensitivity test (14), and serial intravenous injections of norepinephrine before drug pretreatment and $60 \mathrm{~min}$ after fluoroacetate administration.

Finally, fluoroacetate $(2 \mathrm{mg} / \mathrm{kg})$ was administered to six conscious dogs to avoid the possible effects of anesthetic agents on the cardiac output response to fluoroacetate. Blood flows to various organs were also studied by the radioactive microsphere method of Rudolph and Heymann (15). The animals were sedated with morphine sulfate $(1 \mathrm{mg} / \mathrm{kg}$ subcutaneously) and instrumented for measuring cardiac output as previously described for the anesthetized dogs, except their vessels were cannulated under local lidocaine (Xylocaine) anesthesia. Organ blood flows were measured before and $30 \mathrm{~min}$ after fluoroacetate administration. NEN-TRAC microspheres (New England Nuclear, Boston, Mass.), 15 \pm 3 $\mu \mathrm{m}$ in diameter and labeled with either cobalt-57 or 

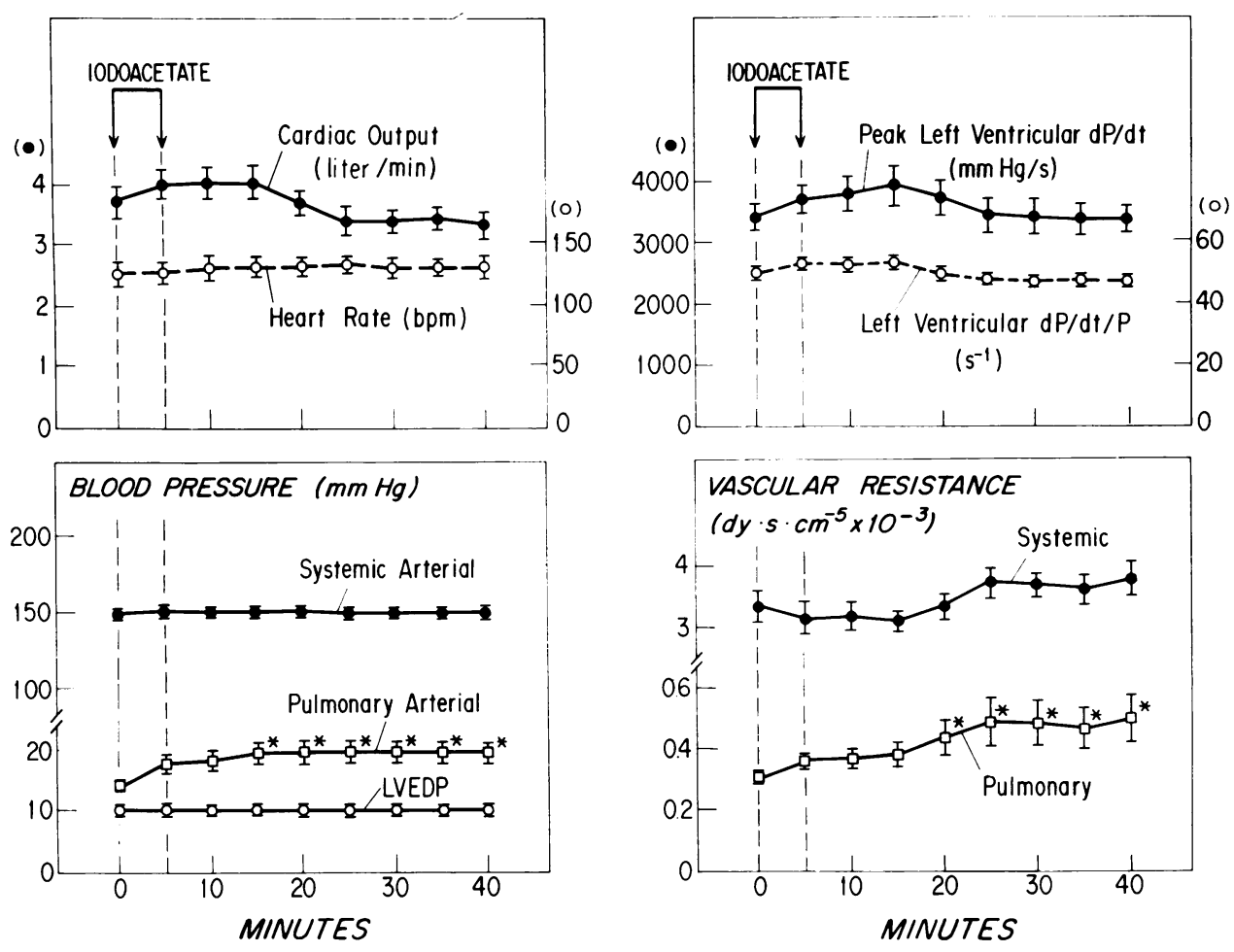

FIGURE 2 Systemic and pulmonary hemodynamic effects of iodoacetate in eight dogs. Vertical lines indicate the means \pm SE. LVEDP, left ventricular end-diastolic pressure; bpm, beats per minute. Asterisks indicate values that are different from the control at $P<0.05$.

manganese-54 at a specific activity of $10 \mathrm{mCi} / \mathrm{g}$, were used. To determine organ blood flows, 450,000 microspheres were injected into the left ventricle via a catheter, which was immediately flushed with $10 \mathrm{ml}$ of normal saline over a 30 s period. Arterial reference blood was withdrawn with a Harvard pump (Harvard Apparatus Co., Inc., Millis, Mass.) at a rate of $7.75 \mathrm{ml} / \mathrm{min}$, beginning $10 \mathrm{~s}$ before the injection of microspheres and continuing for $80 \mathrm{~s}$ thereafter. At the end of the experiment, the dog was sacrificed with intravenous sodium pentobarbital; the lungs, heart, brain, kidneys, liver, stomach, small intestine, large intestine, spleen, pancreas, urinary bladder, ureters, adrenal glands, skeletal muscle, femur, and skin were removed, cleaned, and weighed. After the organs had been ashed in an oven, their content of radioactivity was measured in a Packard gamma spectrometer (Packard Instrument Co., Inc., Downers Grove, Ill.) at the appropriate gamma photon energy for each of the two radionuclides. Absolute blood flow to each organ and tissue was calculated on a PDP-11/10 minicomputer (Digital Equipment Corp., Marlboro, Mass.) by the reference sample method (16) as follows: Organ flow $(\mathrm{ml} / 100 \mathrm{~g}$ per $\mathrm{min})=$ Arterial reference flow $(\mathrm{ml} / \mathrm{min}) \times$ organ nuclide activity $\times 100 /$ Arterial reference nuclide activity $x$ organ weight $(\mathrm{g})$.

Experimental results were analyzed by analysis of variance for repeated measures (17) and the statistical significance of differences between the control and experimental values was determined by Dunnett's test (18). The changes are considered statistically significant if $P$ values are $<0.05$. Student's $t$ test was used to determine the statistical significance of a difference between two means. Values given in Results are mean $\pm \mathrm{SE}$.

\section{RESULTS}

Glycolytic blockade by iodoacetate. Iodoacetate increased concentrations of fructose-1,6-diphosphate, glyceraldehyde-3-phosphate, and dihydroxyacetone phosphate in arterial whole blood and skeletal muscle (Fig. 1). Both pulmonary arterial blood pressure and vascular resistance increased (Fig. 2), but neither systemic nor coronary hemodynamics changed significantly (Fig. 2, Table I). Furthermore, iodoacetate infusion did not change total body oxygen consumption significantly $(126 \pm 5-136 \pm 9 \mathrm{ml} / \mathrm{min})$.

Cardiac metabolism was not affected significantly by iodoacetate, as determined by myocardial extraction coefficients of oxygen, lactate, glucose, and free fatty acids (Table II). The myocardial extraction coefficient of pyruvate was slightly reduced and arterial glucose concentration increased. Arterial concentrations of lactate, pyruvate, and free fatty acids did not change significantly. None of the rates of mycoardial utilization of lactate, pyruvate, glucose, and free fatty acids changed significantly. In addition, neither arterial nor coronary sinus blood $\mathrm{pH}$ changed.

Systemic and pulmonary hemodynamics did not change significantly after administration of normal saline. Cardiac output was $3.31 \pm 0.30,3.29 \pm 0.31$, and 
TABLE I

Effects of Iodoacetate, Fluoroacetate, and Normal Saline on Coronary Hemodynamics

\begin{tabular}{|c|c|c|c|c|c|c|c|}
\hline & CBF & $\mathrm{MV்O}_{2}$ & $\mathrm{C}_{\mathrm{cs}} \mathrm{O}_{2}$ & $\mathrm{O}_{2}$ ext & CVRd & LVW & ME \\
\hline & $m l / 100$ & $m l / 100$ & & & $d y n \cdot s \cdot \mathrm{cm}^{-5}$ & & \\
\hline & $g / \min$ & $g / \min$ & ml/liter & $\%$ & $\times 10^{-3}$ & $\mathrm{~kg} \cdot \mathrm{m} / \mathrm{min}$ & $\%$ \\
\hline \multicolumn{8}{|c|}{ Iodoacetate $(n=8,21.9 \pm 1.2 \mathrm{~kg})$} \\
\hline Control & $89 \pm 6$ & $15.0 \pm 1.0$ & $60 \pm 8$ & $74 \pm 4$ & $74.6 \pm 8.0$ & $9.3 \pm 0.8$ & $30.4 \pm 1.7$ \\
\hline $20 \mathrm{~min}$ & $90 \pm 5$ & $15.7 \pm 1.1$ & $70 \pm 10$ & $71 \pm 4$ & $72.5 \pm 5.6$ & $9.2 \pm 0.6$ & $29.2 \pm 2.0$ \\
\hline $40 \mathrm{~min}$ & $86 \pm 5$ & $15.1 \pm 0.6$ & $66 \pm 7$ & $73 \pm 3$ & $76.3 \pm 7.9$ & $8.5 \pm 0.6$ & $27.6 \pm 1.8$ \\
\hline \multicolumn{8}{|c|}{ Fluoroacetate $(n=14,21.2 \pm 1.1 \mathrm{~kg})$} \\
\hline Control & $99 \pm 7$ & $14.2 \pm 1.3$ & $58 \pm 3$ & $74 \pm 2$ & $61.1 \pm 5.8$ & $8.2 \pm 0.7$ & $25.4 \pm 2.3$ \\
\hline $30 \mathrm{~min}$ & $472 \pm 62^{*}$ & $15.4 \pm 2.0$ & $203 \pm 9^{*}$ & $17 \pm 2 *$ & $12.9 \pm 2.0^{*}$ & $9.2 \pm 0.8$ & $30.8 \pm 4.2$ \\
\hline $60 \mathrm{~min}$ & $419 \pm 33^{*}$ & $14.6 \pm 1.7$ & $203 \pm 9 *$ & $17 \pm 2 *$ & $12.4 \pm 1.6^{*}$ & $8.3 \pm 0.6$ & $26.4 \pm 2.5$ \\
\hline \multicolumn{8}{|c|}{ Normal saline $(n=5,20.2 \pm 1.0 \mathrm{~kg})$} \\
\hline Control & $95 \pm 7$ & $15.2 \pm 1.6$ & $61 \pm 8$ & $74 \pm 4$ & $62.9 \pm 8.2$ & $8.7 \pm 1.0$ & $28.4 \pm 3.3$ \\
\hline $20 \min$ & $99 \pm 8$ & $16.1 \pm 1.5$ & $61 \pm 12$ & $73 \pm 3$ & $60.4 \pm 9.0$ & $9.3 \pm 1.1$ & $28.6 \pm 4.0$ \\
\hline $40 \mathrm{~min}$ & $93 \pm 7$ & $14.0 \pm 1.1$ & $66 \pm 8$ & $70 \pm 3$ & $63.2 \pm 7.8$ & $8.9 \pm 0.5$ & $31.1 \pm 3.2$ \\
\hline
\end{tabular}

Values are means $\pm S E$. The numbers of experiments and body weights of the dogs are given in parentheses after each subheading. Asterisks indicate values that are different from the control at $P<0.05$, as determined by Dunnett's test.

Abbreviations are as follows: $\mathrm{CBF}$, coronary blood flow; $\mathrm{C}_{\mathrm{cs}} \mathrm{O}_{2}$, coronary sinus blood oxygen content; CVRd, diastolic coronary vascular resistance; $\mathrm{LVW}$, left ventricular work; $\mathrm{ME}$, index of myocardial efficiency; $\mathrm{MVO}_{2}$, myocardial oxygen consumption; $\mathrm{O}_{2}$ ext, myocardial oxygen extraction.

TABLE II

Effects of Iodoacetate, Fluoroacetate, and Normal Saline on Cardiac Metabolism

\begin{tabular}{|c|c|c|c|c|c|c|c|c|c|c|c|c|c|c|}
\hline & & & \multicolumn{3}{|c|}{ Lactate } & \multicolumn{3}{|c|}{ Pyruvate } & \multicolumn{3}{|c|}{ Glucose } & \multicolumn{3}{|c|}{ FFA } \\
\hline & \multicolumn{2}{|c|}{$\mathrm{pH}$} & \multirow[b]{2}{*}{ Art } & \multirow{2}{*}{$\begin{array}{l}\text { Ex- } \\
\text { trac- } \\
\text { tion }\end{array}$} & \multirow{2}{*}{$\begin{array}{l}\text { Uti- } \\
\text { liza- } \\
\text { tion }\end{array}$} & \multirow[b]{2}{*}{ Art } & \multirow{2}{*}{$\begin{array}{l}\text { Ex- } \\
\text { trac- } \\
\text { tion }\end{array}$} & \multirow{2}{*}{$\begin{array}{l}\text { Uti- } \\
\text { liza- } \\
\text { tion }\end{array}$} & \multirow[b]{2}{*}{ Art } & \multirow{2}{*}{$\begin{array}{c}\text { Ex- } \\
\text { trac- } \\
\text { tion }\end{array}$} & \multirow{2}{*}{$\begin{array}{l}\text { Uti- } \\
\text { liza- } \\
\text { tion }\end{array}$} & & \multirow{2}{*}{$\begin{array}{l}\text { Ex- } \\
\text { trac- } \\
\text { tion }\end{array}$} & \multirow{2}{*}{$\begin{array}{l}\text { Uti- } \\
\text { liza- } \\
\text { tion }\end{array}$} \\
\hline & Art & cs & & & & & & & & & & Art & & \\
\hline & & & $\begin{array}{c}\mu m o l / \\
m l\end{array}$ & $\%$ & $\begin{array}{c}\mu \mathrm{mol} / \\
100 \mathrm{~g} / \\
\min \end{array}$ & $\begin{array}{c}\mu m o l l \\
m l\end{array}$ & $\%$ & $\begin{array}{c}\mu \mathrm{mol} / \\
100 \mathrm{~g} / \\
\min \end{array}$ & $\begin{array}{c}\mu m o l l \\
m l\end{array}$ & $\%$ & $\begin{array}{c}\text { umoll } \\
100 \mathrm{~g} / \\
\min \end{array}$ & $\begin{array}{c}n m o l l \\
m l\end{array}$ & $\%$ & $\begin{array}{c}\mu \mathrm{mol} / \\
100 \mathrm{~g} / \\
\min \end{array}$ \\
\hline \multicolumn{15}{|c|}{ Iodoacetate } \\
\hline Control & $\begin{array}{r}7.38 \\
\pm 0.02\end{array}$ & $\begin{array}{r}7.29 \\
\pm 0.02\end{array}$ & $\begin{array}{r}2.68 \\
\pm 0.14\end{array}$ & $\begin{array}{r}52.8 \\
\pm 1.9\end{array}$ & $\begin{array}{r}126 \\
\pm 9\end{array}$ & $\begin{array}{r}0.29 \\
\pm 0.02\end{array}$ & $\begin{array}{r}57.1 \\
\pm 2.5\end{array}$ & $\begin{array}{r}15 \\
\pm 2\end{array}$ & $\begin{array}{r}5.15 \\
\pm 0.08\end{array}$ & $\begin{array}{r}1.1 \\
\pm 0.8\end{array}$ & $\begin{array}{r}6 \\
\pm 4\end{array}$ & $\begin{array}{r}389 \\
\pm 36\end{array}$ & $\begin{array}{r}26.7 \\
\pm 5.6\end{array}$ & $\begin{array}{r}9.7 \\
\pm 2.1\end{array}$ \\
\hline $20 \mathrm{~min}$ & $\begin{array}{r}7.41 \\
\pm 0.03\end{array}$ & $\begin{array}{r}7.33 \\
\pm 0.02\end{array}$ & $\begin{array}{r}2.66 \\
\pm 0.33\end{array}$ & $\begin{array}{r}54.4 \\
\pm 3.1\end{array}$ & $\begin{array}{r}130 \\
\pm 19\end{array}$ & $\begin{array}{r}0.30 \\
\pm 0.03\end{array}$ & $\begin{array}{l}48.5 \\
\pm 2.7^{*}\end{array}$ & $\begin{array}{r}13 \\
\pm 2\end{array}$ & $\begin{array}{c}5.99 \\
\pm 0.33^{*}\end{array}$ & $\begin{array}{r}3.9 \\
\pm 0.8\end{array}$ & $\begin{array}{r}20 \\
\pm 4\end{array}$ & $\begin{array}{r}443 \\
\pm 54\end{array}$ & $\begin{array}{r}22.7 \\
\pm 3.6\end{array}$ & $\begin{array}{r}9.4 \\
\pm 2.3\end{array}$ \\
\hline $40 \mathrm{~min}$ & $\begin{array}{r}7.39 \\
\pm 0.02\end{array}$ & $\begin{array}{r}7.32 \\
\pm 0.02\end{array}$ & $\begin{array}{r}2.70 \\
\pm 0.30\end{array}$ & $\begin{array}{r}51.5 \\
\pm 2.6\end{array}$ & $\begin{array}{r}117 \\
\pm 15\end{array}$ & $\begin{array}{r}0.30 \\
\pm 0.02\end{array}$ & $\begin{array}{l}44.8 \\
\pm 4.0^{*}\end{array}$ & $\begin{array}{r}12 \\
\pm 2\end{array}$ & $\begin{array}{c}6.02 \\
\pm 0.36^{*}\end{array}$ & $\begin{array}{r}1.8 \\
\pm 1.5\end{array}$ & $\begin{array}{r}11 \\
\pm 7\end{array}$ & $\begin{array}{r}434 \\
\pm 41\end{array}$ & $\begin{array}{l}20.8 \\
\pm 3.2\end{array}$ & $\begin{array}{r}7.8 \\
\pm 1.4\end{array}$ \\
\hline Fluoroacet & & & & & & & & & & & & & & \\
\hline Control & $\begin{array}{r}7.36 \\
\pm 0.02\end{array}$ & $\begin{array}{r}7.28 \\
\pm 0.01\end{array}$ & $\begin{array}{r}2.10 \\
\pm 0.22\end{array}$ & $\begin{array}{r}50.3 \\
\pm 3.7\end{array}$ & $\begin{array}{r}96 \\
\pm 11\end{array}$ & $\begin{array}{r}0.24 \\
\pm 0.02\end{array}$ & $\begin{array}{r}52.5 \\
\pm 2.8\end{array}$ & $\begin{array}{r}12 \\
\pm 2\end{array}$ & $\begin{array}{r}4.87 \\
\pm 0.23\end{array}$ & $\begin{array}{r}3.0 \\
\pm 0.9\end{array}$ & $\begin{array}{r}14 \\
\pm 4\end{array}$ & $\begin{array}{r}390 \\
\pm 53\end{array}$ & $\begin{array}{r}31.8 \\
\pm 3.5\end{array}$ & $\begin{array}{r}12.7 \\
\pm 2.7\end{array}$ \\
\hline $30 \mathrm{~min}$ & $\begin{array}{r}7.35 \\
\pm 0.01\end{array}$ & $\begin{array}{c}7.30 \\
\pm 0.01^{*}\end{array}$ & $\begin{array}{r}2.33 \\
\pm 0.28\end{array}$ & $\begin{array}{c}18.4 \\
\pm 2.2^{*}\end{array}$ & $\begin{array}{l}177 \\
\pm 22 *\end{array}$ & $\begin{array}{r}0.27 \\
\pm 0.02\end{array}$ & $\begin{array}{l}11.9 \\
\pm 3.7^{*}\end{array}$ & $\begin{array}{r}15 \\
\pm 2\end{array}$ & $\begin{array}{r}4.93 \\
\pm 0.21\end{array}$ & $\begin{array}{c}0.9 \\
\pm 0.8^{*}\end{array}$ & $\begin{array}{r}10 \\
\pm 15\end{array}$ & $\begin{array}{l}312 \\
\pm 48^{*}\end{array}$ & $\begin{array}{l}-5.3 \\
\pm 3.5^{*}\end{array}$ & $\begin{array}{l}-6.8 \\
\pm 5.7^{*}\end{array}$ \\
\hline $60 \mathrm{~min}$ & $\begin{array}{r}7.38 \\
\pm 0.02\end{array}$ & $\begin{array}{c}7.33 \\
\pm 0.01^{*}\end{array}$ & $\begin{array}{c}3.40 \\
\pm 0.30 *\end{array}$ & $\begin{array}{l}11.8 \\
\pm 1.8^{*}\end{array}$ & $\begin{array}{l}167 \\
\pm 23^{*}\end{array}$ & $\begin{array}{r}0.29 \\
\pm 0.02\end{array}$ & $\begin{array}{r}8.9 \\
\pm 1.7^{*}\end{array}$ & $\begin{array}{r}11 \\
\pm 2\end{array}$ & $\begin{array}{r}4.83 \\
\pm 0.28\end{array}$ & $\begin{array}{l}-0.2 \\
\pm 0.4^{*}\end{array}$ & $\begin{array}{l}-1 \\
\pm 9\end{array}$ & $\begin{array}{l}342 \\
\pm 50^{*}\end{array}$ & $\begin{array}{c}0.8 \\
\pm 3.5^{*}\end{array}$ & $\begin{array}{r}3.2 \\
\pm 6.1\end{array}$ \\
\hline $\begin{array}{c}\text { Normal sal } \\
\text { Control }\end{array}$ & $\begin{array}{r}7.36 \\
\pm 0.02\end{array}$ & $\begin{array}{r}7.29 \\
\pm 0.01\end{array}$ & $\begin{array}{r}2.06 \\
\pm 0.26\end{array}$ & $\begin{array}{r}50.4 \\
\pm 4.4\end{array}$ & $\begin{array}{r}99 \\
\pm 11\end{array}$ & $\begin{array}{r}0.26 \\
\pm 0.03\end{array}$ & $\begin{array}{r}48.9 \\
\pm 6.1\end{array}$ & $\begin{array}{r}12 \\
\pm 2\end{array}$ & $\begin{array}{r}5.15 \\
\pm 0.26\end{array}$ & $\begin{array}{r}3.7 \\
\pm 0.6\end{array}$ & $\begin{array}{r}18 \\
\pm 5\end{array}$ & $\begin{array}{r}371 \\
\pm 48\end{array}$ & $\begin{array}{r}29.3 \\
\pm 3.1\end{array}$ & $\begin{array}{r}10.3 \\
\pm 1.5\end{array}$ \\
\hline $20 \mathrm{~min}$ & $\begin{array}{r}7.37 \\
\pm 0.02\end{array}$ & $\begin{array}{r}7.28 \\
\pm 0.01\end{array}$ & $\begin{array}{r}2.04 \\
\pm 0.47\end{array}$ & $\begin{array}{r}48.2 \\
\pm 3.0\end{array}$ & $\begin{array}{r}97 \\
\pm 9\end{array}$ & $\begin{array}{r}0.26 \\
\pm 0.05\end{array}$ & $\begin{array}{r}41.5 \\
\pm 5.0\end{array}$ & $\begin{array}{r}11 \\
\pm 1\end{array}$ & $\begin{array}{r}4.86 \\
\pm 0.22\end{array}$ & $\begin{array}{r}3.7 \\
\pm 1.2\end{array}$ & $\begin{array}{r}17 \\
\pm 4\end{array}$ & $\begin{array}{r}374 \\
\pm 70\end{array}$ & $\begin{array}{r}33.4 \\
\pm 4.2\end{array}$ & $\begin{array}{r}12.6 \\
\pm 2.0\end{array}$ \\
\hline $40 \mathrm{~min}$ & $\begin{array}{r}7.36 \\
\pm 0.02\end{array}$ & $\begin{array}{r}7.29 \\
\pm 0.02\end{array}$ & $\begin{array}{r}1.95 \\
\pm 0.50\end{array}$ & $\begin{array}{r}50.4 \\
\pm 3.4\end{array}$ & $\begin{array}{r}93 \\
\pm 9\end{array}$ & $\begin{array}{r}0.25 \\
\pm 0.03\end{array}$ & $\begin{array}{r}42.5 \\
\pm 5.2\end{array}$ & $\begin{array}{r}10 \\
\pm 2\end{array}$ & $\begin{array}{r}4.92 \\
\pm 0.30\end{array}$ & $\begin{array}{r}2.3 \\
\pm 0.8\end{array}$ & $\begin{array}{r}11 \\
\pm 7\end{array}$ & $\begin{array}{c}341 \\
\pm 51\end{array}$ & $\begin{array}{l}28.8 \\
\pm 4.5\end{array}$ & $\begin{array}{r}10.0 \\
\pm 1.4\end{array}$ \\
\hline
\end{tabular}

Values are means \pm SE. The number of experiments and body weights of the dogs are the same as in Table I. Asterisks indicate values that are different from the control at $P<0.05$, as determined by Dunnett's test.

Art and Cs indicate arterial blood and coronary sinus venous blood, respectively. Arterial blood concentrations of lactate, pyruvate, and glucose, and arterial plasma concentrations of free fatty acids (FFA) are given. 
$3.29 \pm 0.23$ liters $/ \mathrm{min}$, and pulmonary arterial blood pressure was $14.7 \pm 0.7,14.1 \pm 0.4$, and $14.5 \pm 0.4 \mathrm{~mm} \mathrm{Hg}$, at 0,20 , and $40 \mathrm{~min}$, respectively, after normal saline administration. Normal saline administration also did not result in significant change in coronary hemodynamics (Table I) or cardiac metabolism (Table II).

Tricarboxylic acid cycle inhibition by fuoroacetate. Blood and skeletal muscle citrate concentrations increased after fluoroacetate administration (Fig. 3). Likewise, cardiac citrate concentration $60 \mathrm{~min}$ after fluoroacetate administration $(0.44 \pm 0.05 \mu \mathrm{mol} / \mathrm{g}$ fresh weight, $n=7$ ) was significantly higher than the control value $(0.27 \pm 0.02 \mu \mathrm{mol} / \mathrm{g}$ fresh weight, $n=5)$.

Like iodoacetate, fluoroacetate produced increases in pulmonary arterial blood pressure and vascular resistance, and had no significant effects on total body oxygen consumption (124 $\pm 5-124 \pm 6 \mathrm{ml} / \mathrm{min})$ and systemic hemodynamic parameters, except for a small decrease in systemic arterial blood pressure at $1 \mathrm{~h}$ (Fig. 4).

On the other hand, coronary blood flow markedly increased after administration of fluoroacetate, accompanied by a reduction in diastolic coronary vascular resistance (Table I, Fig. 5). Coronary sinus oxygen content increased to $350 \%$ of the control with a marked reduction in myocardial oxygen extraction (Table $I$ ), but myocardial oxygen consumption did not change (Table I, Fig. 5). In addition, neither left ventricular work nor the index of myocardial efficiency changed significantly (Table I).

Cardiac metabolism was markedly influenced by fluoroacetate (Table II). Coronary sinus blood pH increased, but arterial blood $\mathrm{pH}$ did not change significantly. Fluoroacetate increased arterial blood lactate concentration and decreased arterial plasma free fatty acid concentration. The myocardial extraction coefficients of lactate, pyruvate, glucose, and free fatty acids all fell, but the rate of myocardial utilization of lactate increased. There was no change in myocardial utilization of pyruvate and glucose, but the rate of myocardial utilization of free fatty acids was reduced. Coronary sinus plasma calcium concentration, measured in nine dogs, did not change significantly after fluoroacetate administration (8.8 $\pm 0.5-9.0 \pm 0.4$ $\mathrm{mg} / 100 \mathrm{ml}$ ).

Effects of autonomic nervous system inhibition on hemodynamic responses to fluoroacetate administration. Mecamylamine pretreatment reduced the pressor response to common carotid occlusion from $53 \pm 9$ to $3 \pm 1 \mathrm{~mm} \mathrm{Hg}(n=5, P<0.01)$. Propranolol pretreatment increased the dose of isoproterenol required to produce a rise in heart rate of 25 beats $/ \mathrm{min}$ from $1.6 \pm 0.2$ to $30.0 \pm 4.1 \mu \mathrm{g}(n=10, P<0.001)$. Phentolamine pretreatment, on the other hand, increased the dose of norepinephrine needed to raise mean systemic

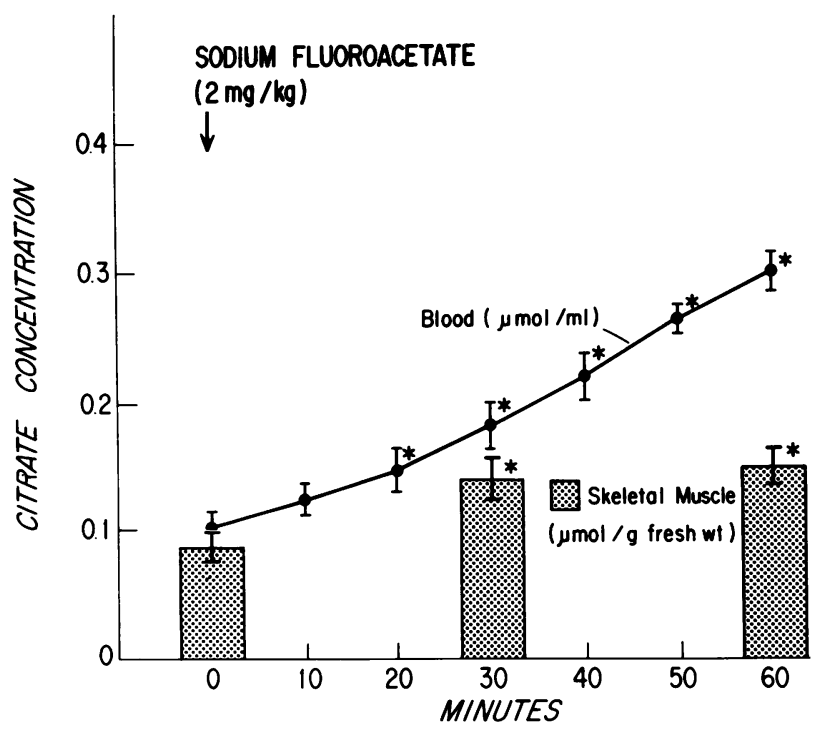

FIGURE 3 Changes in arterial blood $(n=14)$ and skeletal muscle $(n=6)$ citrate concentration after fluroacetate administration. Vertical lines indicate the means \pm SE. Asterisks indicate values that are different from the control at $P$ $<0.05$.

arterial blood pressure by $25 \mathrm{~mm} \mathrm{Hg}$ from $2.5 \pm 0.6$ to $25.9 \pm 4.5 \mu \mathrm{g}(\mathrm{n}=5, P<0.01)$.

In these animals $(21.9 \pm 1.0 \mathrm{~kg})$ after autonomic nervous system inhibition, fluoroacetate administration did not cause significant systemic hemodynamic changes, but still produced increases in pulmonary arterial blood pressure and coronary blood flow as it did in intact dogs. Pulmonary arterial blood pressure increased from $13.3 \pm 0.7$ to $16.1 \pm 1.1$ and $17.7 \pm 1.1$ $\mathrm{mm} \mathrm{Hg}$, and coronary blood flow from $61 \pm 5$ to $243 \pm 48$ and $198 \pm 26 \mathrm{ml} / 100 \mathrm{~g}$ per min, at 30 and $60 \mathrm{~min}$, respectively, after fluoroacetate administration. All of these changes from control values are statistically significant at $P<0.05$.

Effects of fluoroacetate on organ blood flows. Table III shows the changes in organ blood flows after fluoroacetate administration in six conscious dogs $(9.6 \pm 1.0 \mathrm{~kg})$. Myocardial blood flow increased and hepatic artery flow fell, whereas other organ flows did not change significantly. As in anesthetized dogs, there were no changes in cardiac output $(1.65 \pm 0.25$ $1.76 \pm 0.21$ liters $/ \mathrm{min})$ or mean systemic arterial blood pressure $(120 \pm 6-121 \pm 5 \mathrm{~mm} \mathrm{Hg})$.

\section{DISCUSSION}

Significant metabolic inhibition was produced by iodoacetate and fluoroacetate in the present experiments. In addition to phosphoglyceraldehyde dehydrogenase, iodoacetate also inhibits a variety of other enzymes containing thiol groups; anaerobic glycolysis, however, is inhibited by iodoacetate to a much greater extent 

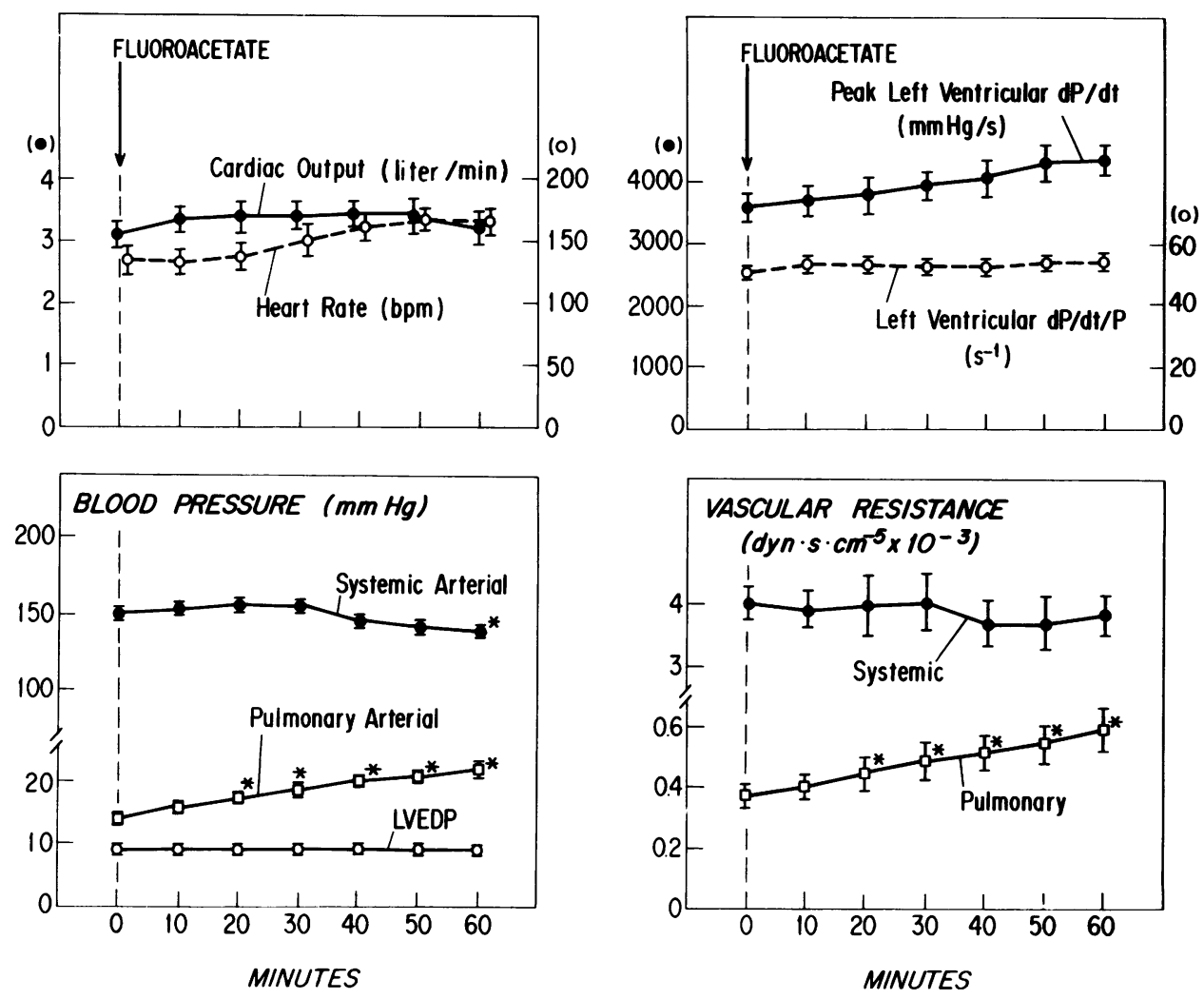

FIGURE 4 Systemic and pulmonary hemodynamic effects of fluoroacetate in 14 dogs. Vertical lines indicate the means $\pm \mathrm{SE}$. Asterisks indicate values that are different from the control at $P<0.05$. For abbreviations see Fig. 2 .

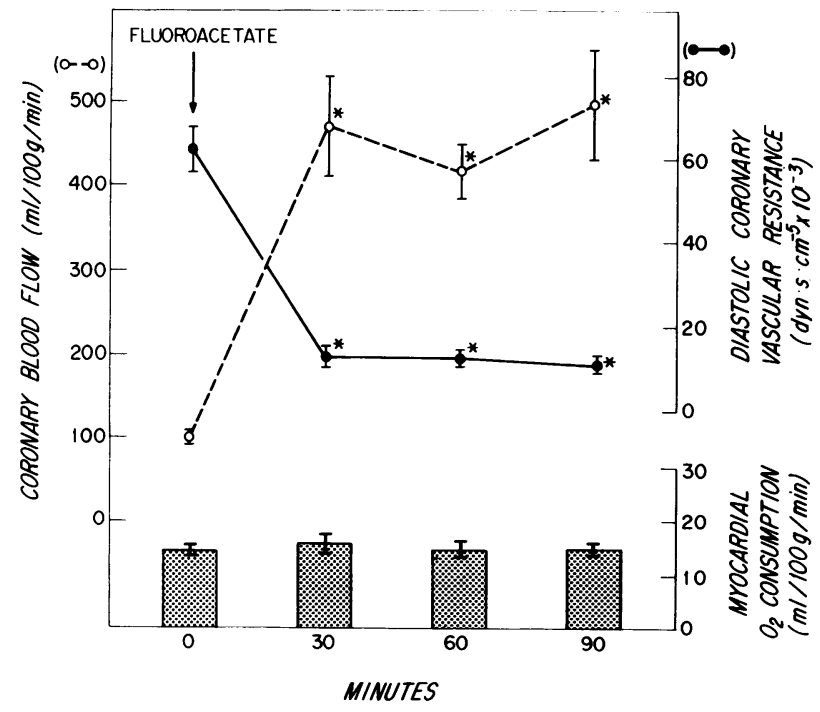

FIGURE 5 Changes in coronary blood flow, diastolic coronary vascular resistance, and myocardial oxygen consumption after fluoroacetate administration in 14 experiments. $n=6$ for values at $90 \mathrm{~min}$ after fluoroacetate administration. Vertical lines indicate the means $\pm S E$. Asterisks indicate values that are different from the control at $P<0.05$. than aerobic respiration (19). If iodoacetate is distributed evenly within the body, its concentration achievable in the present experiments would not have exceeded $0.3 \mathrm{mM}$, a concentration at which iodoacetate exerts a rather specific action on glycolysis (19). The present results suggest that iodoacetate exerted no significant actions on the tricarboxylic acid cycle and on the electron transport system of the myocardium.

Fluoroacetate condenses with oxaloacetate in vivo to produce fluorocitrate which selectively and competitively inhibits citrate utilization by its action on aconitase (20). At very large doses, fluorocitrate inhibits succinate dehydrogenase (21), but it has no direct action on other enzymes or on oxidative phosphorylation (20).

Arterial lactate concentration increased and myocardial utilization of free fatty acids was abolished after fluoroacetate administration. Similarly, it is known that a shift from fatty acid oxidation to increased glycolysis occurs during hypoxia (22-24), probably partly because of the highly accelerated activity of phosphofructokinase (23) relative to that of thiokinase. However, neither total body nor myocardial oxygen 
consumption changes significantly in intact animals during mild to moderately severe hypoxia (25-28), partly because the increased flow compensates for the reduction in arterial oxygen content (26). Likewise, oxygen consumption did not change after fluoroacetate administration in the present experiments. The partial inhibitory action of fluoroacetate probably was offset by the increased citrate concentration (29). The heart also may utilize glutamate and aspartate, which feed into the tricarboxylic acid cycle distal to the aconitase step. Administration of larger doses $(4 \mathrm{mg} / \mathrm{kg}$ ) of fluoroacetate caused periodic convulsion, lactic acidosis, and an increase in oxygen consumption (unpublished observation), making it difficult to interpret the hemodynamic changes.

A pulmonary pressor response to iodoacetate has been reported in isolated perfused lungs $(30,31)$, indicating a direct action of iodoacetate on the lung. The present study demonstrates that both iodoacetate and fluoroacetate cause pulmonary vasoconstriction, and that the pulmonary pressor effect of fluoroacetate is not mediated by adrenergic mechanism.

The experiments with iodoacetate show that pulmonary vasoconstriction is correlated with the accumulation of glycolytic metabolites, in the absence of reduced oxygen tension. Reduced production of ATP via the glycolytic pathway is expected, but aerobic metabolism and oxidative phosphorylation were not altered. These changes in glycolytic metabolites, which also occur during hypoxia $(22,23,32)$ and after fluoroacetate administration (29), may be responsible directly or indirectly for the pulmonary pressor response.

Coronary blood flow increased and diastolic coronary vascular resistance decreased after fluoroacetate administration. Iodoacetate administration, however, had no effects on coronary circulation. The heart possesses a rapid metabolic rate, great dependence on aerobic metabolism (33), and greater reliance on autoregulation of the flow than on neural control (34). Whether fluoroacetate also increases the blood flow to exercising skeletal muscle which exhibits the same metabolic properties warrants further study. The coronary vascular effects of fluoroacetae probably were not mediated by the autonomic nerves or adrenergic neurohumors to the heart (35), because systemic hemodynamics and myocardial contractility did not change significantly and because vagotomy and sympathetic blockade did not abolish this coronary dilator response to fluoroacetate. The reduction in coronary blood flow after autonomic nervous system inhibition is consistent with the finding that coronary blood flow decreases after surgical cardiac denervation to one-half the value obtained in the normal dog (36), indicating that the denervated heart functions at a lower metabolic rate than the heart with normal innervation.

Coronary flow increased after addition of fluoroace-
TABLE III

Organ Blood Flows before and 30 Min after Fluoroacetate Administration in Six Conscious Dogs

\begin{tabular}{lccc}
\hline & & \multicolumn{2}{c}{ Blood flow } \\
\cline { 3 - 4 } \multicolumn{1}{c}{ Organ } & Weight & Control & Fluoroacetate \\
\hline & $g$ & \multicolumn{2}{c}{$m l / 100$ g/min } \\
Left ventricle & $51 \pm 6$ & $80 \pm 8$ & $523 \pm 47^{*}$ \\
Right ventricle & $20 \pm 1$ & $53 \pm 7$ & $299 \pm 45^{*}$ \\
Brain & $66 \pm 2$ & $35 \pm 7$ & $62 \pm 22$ \\
Lungs (bronchial artery) & $115 \pm 16$ & $32 \pm 4$ & $49 \pm 13$ \\
Kidneys & $67 \pm 5$ & $221 \pm 23$ & $274 \pm 47$ \\
Adrenals & $1.3 \pm 0.1$ & $206 \pm 22$ & $343 \pm 80$ \\
Liver (hepatic artery) & $300 \pm 33$ & $77 \pm 12$ & $35 \pm 13 *$ \\
Spleen & $27 \pm 5$ & $138 \pm 24$ & $141 \pm 45$ \\
Pancreas & $29 \pm 3$ & $28 \pm 4$ & $38 \pm 9$ \\
Urinary bladder and & & & \\
$\quad$ ureters & $13 \pm 2$ & $10 \pm 2$ & $11 \pm 3$ \\
Stomach & $149 \pm 12$ & $16 \pm 2$ & $15 \pm 2$ \\
Small intestine & $314 \pm 19$ & $27 \pm 3$ & $32 \pm 5$ \\
Large intestine & $61 \pm 8$ & $37 \pm 8$ & $34 \pm 7$ \\
Skin (leg) & & $7 \pm 2$ & $3 \pm 1$ \\
Muscle (leg) & & $4 \pm 1$ & $3 \pm 1$ \\
Bone (femur) & & $11 \pm 2$ & $6 \pm 2$ \\
\hline
\end{tabular}

Values are means \pm SE. Asterisks indicate values that are significantly different from the control at $P<0.05$, as determined by Student's $t$ test for paired data.

tate to isolated perfused hearts (29). In addition, coronary sinus blood oxygen content increased. It appears that these coronary vascular effects of fluoroacetate were associated with metabolic changes occurring between the sites of action of iodoacetate and fluoroacetate, or with a reduction in high energy phosphate production (29). It was not caused by the calcium-chelating effect of citrate because neither the coronary sinus plasma calcium concentration in the dog nor the total calcium content of the rat heart (37) changed significantly. It also seems unlikely that the control of coronary blood flow was via a change in cytoplasmic redox potential because there was no correlation between coronary blood flow and the blood lactate to pyruvate ratio.

Blood flow to organs other than the heart did not increase significantly after fluoroacetate administration. This cannot be attributed to failure of this agent to act upon tissues other than the heart, because skeletal muscle citrate levels increased. In addition, fluoroacetate is distributed uniformly in the body (38), causing a large accumulation of citrate in almost all tissues $(20,37)$. The kidney exhibits the highest citrate concentration in the body, whereas the citrate level in liver changes only slightly (20). Renal blood flow did not change significantly $30 \mathrm{~min}$ after fluoroacetate administration. Farah, et al. (39), by direct measurements with flowmeters, also found that renal 
blood flow changed relatively little in the first 30 min, but fell to $40-70 \%$ of the control $2-3 \mathrm{~h}$ later. Hepatic arterial blood flow decreased. Similarly, it decreases during severe hypoxia, probably a result of adrenergic stimulation (40).

The increases in cardiac output and myocardial contractility during hypoxia probably are related to autoregulation of the peripheral vascular beds (41) and stimulation of the sympathetic nervous system $(1,28,42$, 43). Results of the present study suggest that the systemic hemodynamic effects of hypoxia probably are not causally related to the accumulation of glycolytic metabolites or of other metabolites which are also affected by fluoroacetate. It also appears that the type and degree of the vascular responses are determined, at least in part, by the qualitative and quantitative differences in cellular enzymatic composition that exist in various organs. Further work is needed to determine the role of metabolically linked vasoactive substances (44-46) in the regulation of cardiac output and organ blood flows.

Note added in proof. Additional experiments were performed in collaboration with Dr. John M. Lowenstein to study the effects of fluoroacetate $(2 \mathrm{mg} / \mathrm{kg}$ i.v. $)$ on coronary sinus plasma and myocardial concentrations of adenosine, inosine, and hypoxanthine in five chloralose-anesthetized dogs. The purine nucleosides and hypoxanthine were measured as previously described by Schultz and Lowenstein (47). Coronary sinus plasma concentrations of adenosine, inosine, and hypoxanthine were $0.12 \pm 0.05,0.10 \pm 0.06$, and $0.75 \pm 0.23 \mu \mathrm{mol} /$ iter, respectively, before fluoroacetate administration, and were $0.11 \pm 0.05,0.47 \pm 0.21$, and $3.56 \pm 1.36$ $\mu \mathrm{mol} /$ /iter, respectively, $30 \mathrm{~min}$ after fluoroacetate administration. The changes in inosine and hypoxanthine concentrations were statistically significant at $P<0.05$, as determined by the Student's $t$ test for paired data. Simultaneously, myocardial adenosine concentrations increased to $444 \pm 45 \%$ of the control values. These results suggest that the increase in coronary blood flow produced by fluoroacetate probably was caused by the vasodilator action of adenosine released from the myocardium.

\section{ACKNOWLEDGMENTS}

The author is indebted to Dr. William B. Hood, Jr., for his invaluable advice and criticisms throughout the study, to Dr. John M. Lowenstein of Brandeis University for his careful review of the manuscript, and to Dr. Dieter $M$. Kramsch for measuring plasma calcium concentration. The expert assistance from Adele Rymut, Aase Frederiksen, Deborah Walker, and Charles Scheller is gratefully acknowledged. The following chemicals were generously supplied by pharmaceutical companies: indocyanine green (Cardio-Green) by Hynson, Westcott \& Dunning, Inc., Baltimore, Md.; mecamylamine $\mathrm{HCl}$ (Inversine) by Merck Sharp \& Dohme, Inc., Division of Merck \& Co., Inc., West Point, Pa.; phentolamine $\mathrm{HCl}$ (Regitine) by CIBA Pharmaceutical Co., Summit, N. J.; and propranolol $\mathrm{HCl}$ (Inderal) by Ayerst Research Laboratories, Montreal, Canada.

This study was supported in part by U. S. Public Health Service grants HL-17403, HL-14646, NO 1 HV 53001, and HL-18318 from the National Heart and Lung Institute.

\section{REFERENCES}

1. Liang, C., and W. E. Huckabee. 1973. Mechanisms regulating the cardiac output response to cyanide infusion, a model of hypoxia. J. Clin. Invest. 52: 3115-3128.

2. Hilton, R., and F. Eichholtz. 1925. The influence of chemical factors on the coronary circulation. J. Physiol. (Lond.). 59: 413-425.

3. Krasnow, N., H. J. Levine, R. J. Wagman, and R. Gorlin. 1963. Coronary blood flow measured by $\mathrm{I}^{131}$ iodoantipyrine. Circ. Res. 12: 58-62.

4. Palmer, W. H., T. Zsoter, W. M. Fam, and M. McGregor. 1963. Measurement of coronary blood flow using 4-aminoantipyrine. Circulation. 28: 782. (Abstr.)

5. Gorlin, R. 1961. Measurement of coronary flow in health and disease. In Modern Trends in Cardiology. A. M. Jones, editor. Paul B. Hoeber, Inc., New York. 191-213.

6. Ramsay, L. H. 1959. Analysis of gas in biological fluids by gas chromatography. Science (Wash. D. C.). 129: 900901.

7. Willis, J. B. 1960. Determination of calcium in blood serum by atomic absorption spectroscopy. Nature (Lond.). 186: 249-250.

8. Friedland, I. M. and L. S. Dietrich. 1961. A rapid enzymic determination of L(+)-lactic acid. Anal. Biochem. 2: 390-392.

9. Huckabee, W. E. 1956. Control of concentration gradients of pyruvate and lactate across cell membranes in blood. J. Appl. Physiol. 9: 163-170.

10. Washko, M. E., and E. W. Rice. 1961. Determination of glucose by an improved enzymatic procedure. Clin. Chem. 7: 542-545.

11. Novak, M. 1965. Colorimetric ultramicro method for the determination of free fatty acids. J. Lipid Res. 6: 431433.

12. Michal, G., and H. O. Beutler. 1974. D-fructose-1,6diphosphate, dihydroxyacetone phosphate and D-glyceraldehyde-3-phosphate. In Methods of Enzymatic Analysis. H. U. Bergmeyer, editor. Academic Press, Inc., New York. 2nd edition. 3: 1314-1319.

13. Dagley, S. 1974. Citrate. UV spectrophotometric determination. In Methods of Enzymatic Analysis. H. U. Bergmeyer, editor. Academic Press, Inc., New York. 2nd edition. 3: $1562-1565$.

14. Cleaveland, C. R., R. E. Rangno, and D. G. Shand 1972. Standardized isoproterenol sensitivity test: effects of sinus arrhythmias, atropine and propranolol. Arch. Intern. Med. 130: 47-52.

15. Rudolph, A. M., and M. A. Heymann. 1967. Circulation of the fetus in utero: methods for studying distribution of blood flow, cardiac output and organ blood flow. Circ. Res. 21: 163-184.

16. Buckberg, G. D., J. C. Luck, D. B. Payne, J. I. E. Hoffman, J. P. Archie, and D. E. Fixler. 1971. Some sources of error in measuring regional blood flow with radioactive microspheres. J. Appl. Physiol. 31: 598-604.

17. Winer, B. J. 1971. Statistical Principles in Experimental Design. McGraw-Hill Book Co., New York. 2nd edition. 261-300.

18. Dunnett, C. W. 1964. New tables for multiple comparison with a control. Biometrics. 20: 482-491.

19. Webb, J. L. 1966. Enzymes and Metabolic Inhibitors. Vol. III. Academic Press, Inc., New York. 1-283.

20. Peters, R. A. 1957. Mechanism of the toxicity of the active constituent of Dichapetalum cymosum and related compounds. Adv. Enzymol. Relat. Areas Mol. Biol. 18: 113-159.

21. Kun, E. 1969. Mechanism of action of fluoro analogs 
of citric acid cycle compounds: an essay on biochemical tissue specificity. In Citric Acid Cycle, Control and Compartmentation. J. M. Lowenstein, editor. Marcel Dekker, Inc., New York. 297-339.

22. Williamson, J. R. 1966. Glycolytic control mechanisms. II. Kinetics of intermediate changes during the aerobicanoxic transition in perfused rat heart. J. Biol. Chem. 241: 5026-5036.

23. Newholme, E. A., and P. J. Randle. 1961. Regulation of glucose uptake by muscle. V. Effects of anoxia, insulin, adrenaline and prolonged starving on concentrations of hexose phosphates in isolated rat diaphragm and perfused isolated rat heart. Biochem. J. 80: 655-662.

24. Opie, L. H. 1972. Metabolic response during impending myocardial infarction. I. Relevance of studies of glucose and fatty acid metabolism in animals. Circulation. 45: 483-490.

25. Hackel, D. B., W. T. Goodale, and J. Kleinerman. 1954. Effects of hypoxia on the myocardial metabolism of intact dogs. Circ. Res. 2: 169-174.

26. Berne, R. M., J. R. Blackmon, and T. H. Gardner. 1957. Hypoxemia and coronary blood flow. J. Clin. Invest. 36: $1101-1106$.

27. Vance, J. P., J. R. Parratt, and I. McA. Ledingham. 1971. The effects of hypoxia on myocardial blood flow and oxygen consumption: negative role of beta adrenoreceptors. Clin. Sci. (Oxf.). 41: 257-273.

28. Liang, C., and W. E. Huckabee. 1973. Effects of splenectomy and beta-adrenoceptor blockade on cardiac output response to acute hypoxemia. J. Clin. Invest. 52: 31293134 .

29. Williamson, J. R. 1967. Glycolytic control mechanisms. III. Effects of iodoacetamide and fluoroacetate on glucose metabolism in the perfused rat heart. J. Biol. Chem. 242: 4476-4485.

30. Duke, H. N., and E. M. Killick. 1952. Pulmonary vasomotor responses of isolated perfused cat lungs to anoxia. J. Physiol. (Lond.). 117: 303-316.

31. Liljestrand, G. 1958. Chemical control of the distribution of the pulmonary blood flow. Acta Physiol. Scand. 44: 216-240.

32. Lowry, O. H., J. V. Passonneau, F. X. Hasselberger, and D. W. Schultz. 1964. Effect of ischemia on known substrates and cofactors on the glycolytic pathway in brain. J. Biol. Chem. 239: 18-30.

33. Opie, L. H. 1968. Metabolism of the heart in health and disease. Am. Heart J. 76 (Part I): 685-698.
34. Berne, R. M. 1975. Myocardial blood flow: metabolic determinants. In The Peripheral Circulations. R. Zelis, editor. Grune \& Stratton Inc., New York. 117-129.

35. Mark, A. L., and F. M. Abboud. 1975. Myocardial blood flow: neurohumoral determinants. In The Peripheral Circulations. R. Zelis, editor. Grune \& Stratton Inc., New York. 95-115.

36. Gregg, D. E., E. M. Khouri, D. E. Donald, H. S. Lowensohn, and S. Pasyk. 1972. Coronary circulation in the conscious dog with cardiac neural ablation. Circ. Res. 31: $129-144$.

37. Peters, R. A. 1952. Croonian Lecture: lethal synthesis. Proc. Roy. Soc. B. 139: 143-170.

38. Hagan, E. C., L. L. Ramsey, and G. Woodward. 1950. Absorption, distribution, and excretion of sodium fluoroacetate (1080) in rats. J. Pharmacol. Exp. Ther. 99: 432434.

39. Farah, A., G. Graham, and F. Koda. 1953. The action of sodium fluoroacetate on the renal tubular transport of para-aminohippurate and glucose in the dog. J. Pharmacol. Exp. Ther. 108: 410-423.

40. Fischer, A., L. Takacs, and G. Molmar. 1960. Hepatic circulation in arterial hypoxia. Acta Med. Acad. Sci. Hung. 16: 61-74.

41. Shephard, A. P., H. J. Granger, E. E. Smith, and A. C. Guyton. 1973. Local control of tissue oxygen delivery and its contribution to the regulation of cardiac output. Am. J. Physiol. 225: 747-755.

42. Richardson, D. W., H. A. Kontos, A. J. Raper, and J. L. Patterson, Jr. 1967. Modification by beta-adrenergic blockade of the circulatory responses to acute hypoxia in man. J. Clin. Invest. 46: 77-85.

43. Korner, P. I., and S. W. White. 1966. Circulatory control in hypoxia by the sympathetic nerves and adrenal medulla. J. Physiol. (Lond.). 184: 272-290.

44. Haddy, F. J., and J. B. Scott. 1968. Metabolically linked vasoactive chemicals in local regulation of blood flow. Physiol. Rev. 48: 688-707.

45. Mellander, S., and B. Johnasson. 1968. Control of resistance, exchange and capacitance functions in the peripheral circulation. Pharmacol. Rev. 20: 117-196.

46. Somlyo, A. P., and A. V. Somlyo. 1970. Vascular smooth muscle. II. Pharmacology of normal and hypertensive vessels. Pharmacol. Rev. 22: 249-353.

47. Schultz, V., and J. M. Lowenstein. 1976. Purine nucleotide cycle. Evidence for the occurrence of the cycle in brain. J. Biol. Chem. 251: 485-492. 